Aus der Chirurgischen Universitäts-Klinik Würzburg. (Vorstand: Geh. Rat Prof. Dr. F. König.)

\title{
Klinische Untersuchungen über das Verhalten von Blutdruck und Puls während und nach Novokain- Suprarenin-Anästhesierung ${ }^{1}$ ).
}

Von Otto Wiemann, Assistent der Klinik.

(Mit II Kurven.)

In mehreren Arbeiten aus letzter Zeit, die sich mit den Nebenwirkungen der neueren Verfahren der Leitungsanästhesie, vor allem auch der Splanchnicusanästhesie befassen, wird auch über Änderungen der Pulsfrequenz und des Blutdrucks berichtet, doch scheint ein sicheres Ergebnis bezüglich der Wirkung der Lokalanästhesie in dieser Beziehung bis jetzt noch nicht gewonnen zu sein. Es wird teils über Blutdrucksteigerung, teils über Blutdrucksenkung während oder nach der Anästhesierung berichtet, da es sich aber zum Teil auch um Fälle handelt, bei denen die Anästhesie oder die Operation keinen ungestörten Verlauf nahm, so ist die Beurteilung der beobachteten Puls- und Blutdruckänderungen bezüglich ihrer Ursache noch besonders erschwert. Ein Rückschluß auf eine normale Kreislaufwirkung des NovokainSuprarenins bei ungestörter Anästhesie und Operation läßt sich aus diesen Beobachtungen nicht gewinnen. Gerade in dieser Beziehung erschien es zweckmäßig, dem Verhalten des Blutdrucks und des Pulses bei einer ungestörten Operation in Lokalanästhesie besondere Aufmerksamkeit zu schenken, wie es für die Allgemeinnarkose schon längst in genauen Untersuchungen geschehen ist.

$\mathrm{Zu}$ diesem $\mathrm{Zweck}$ wurden bei einer Anzahl von Patienten genaue Blutdruckmessungen und Pulszählungen vorgenommen, die sich auf den Zeitraum vor Beginn der Anästhesierung bis zum Schluß der Operation erstreckten, bei Patienten mit Hernien, Hydrocelen, Strumen, Magenerkrankungen, bei denen Novokain-

I) Abgeschlossen Februar $192 \mathbf{I}$. 
Suprarenin teils zur lokalen Infiltration, teils zur Leitungsanästhesie, in sehr verschiedenen Mengen zur Anwendung kam. Es erschien notwendig, die Beobachtung von Puls- und Blutdruck schon einige Minuten vor Anfang der Anästhesierung zu beginnen und die Messungen weiterhin bis zum Schluß der Operation in regelmäßigen Abständen von 2 Minuten zu wiederholen, um über die auftretenden Puls- und Blutdruckänderungen in Form einer Kurve ein deutliches Bild zu gewinnen. In den hier verwerteten Fällen handelt es sich ausschlieBlich, mit Ausnahme der noch besonders erwähnten, um glatt verlaufene Anästhesien, bei denen weder bei der Anästhesierung noch bei der folgenden Operation Zeichen einer allgemeinen Novokain-Suprareninintoxikation, insbesondere Erscheinungen von seiten des Nervensystems, beobachtet wurden, und die bezüglich der Ausschaltung der Schmerzempfindung als gut oder sehr gut bezeichnet werden mußten. Als Vorbereitung zur Operation diente Morphium (bei Frauen o,or, bei Männern 0,02 g), während auf die Verabreichung noch anderer Narkotika zur Erzielung eines Dämmerzustandes verzichtet wurde. Die Kreislaufwirkung der verwandten Morphiumgaben ist so gering, daß sie praktisch vernachlässigt werden kann, abgesehen von den Fällen, wo eine Morphiumintoxikation auftritt. Über die Beurteilung von Blutdruckwerten gibt die interne Literatur Aufschluß, ein großer Teil der Gesichtspunkte, die bei der Beurteilung vom chirurgischen Standpunkt von Wert sind, findet sich auch in den Arbeiten erwähnt, die sich mit der Kreislaufwirkung der Allgemeinnarkose befassen. Für den vorliegenden $Z$ weck handelt es sich nicht darum, den Normalblutdruckwert eines Patienten vor Beginn der Lokalanästhesie zu bestimmen, sondern nur darum, wie sich unter dem Einfluß der Novokain-Suprarenininjektion der Blutdruck und der Puls ändern im Vergleich zu dem vorher festgestellten Wert. Ob dieser letztere dem normalen Blutdruckwert des betreffenden Patienten entspricht, oder durch psychische und andere Einflüsse verändert ist, ist von untergeordneter Bedeutung. Die ganze Beurteilung der beobachteten Blutdruckschwankungen wird erleichtert, wenn schon einige Minuten vor Beginn der Anästhesierung Puls und Blutdruck bei mehrmaligen Messungen einen ziemlich konstanten Wert aufweisen. Der Einfluß psychischer Momente wird durch eine gute Morphiumwirkung sicher verringert. Auch 
sonst lassen sich durch langsames und vorsichtiges Vorgehen bei der Anästhesierung manche Ursachen, die für eine Blutdruckänderung bzw. Änderung der Pulsfrequenz in Frage kommen, vermeiden, z. B. die Unannehmlichkeit des ersten Einstichs der Kanuile. Dadurch, daß nach dem letzteren I-2 Minuten gewartet wird und erst dann die Injektion vorgenommen wird, lassen sich etwaige dadurch bedingte Beeinflussungen von Puls und Blutdruck, die nach unseren Erfahrungen allerdings unwesentlich sind, in der Kurve deutlich erkennen und von den Resorptionswirkungen des Anästhetikums um so sicherer unterscheiden. Zweckmäßig ist es weiterhin, zwischen Vollendung der Anästhesierung und dem Operationsbeginn einen gewissen Zeitraum einzuschalten, der es ermöglicht, Puls und Blutdruck in einer Periode beobachten zu können, in der eine Beeinflussung durch die Operation noch nicht stattfindet. Vielfach wurde I proz. N.S.-Lösung und in größeren Mengen angewandt, als für die notwendige Anästhesie unbedingt erforderlich war, auch die Lösung, mit Ausnahme der besonders erwähnten Fälle, aus den stärker adrenalinhaítigen Tabletten B. hergestellt, in der Absicht, dadurch eine deutlichere Beeinflussung der Puls- und Blutdruckkurve zu erhalten.

In einer Anzahl von Fällen kam es weder während der Anästhesierung noch während der Operation zu wesentlichen Veränderungen von Puls und Blutdruck, als unwesentlich wurde eine Schwankung des Blutdrucks bis zu $20 \mathrm{~mm}$ Quecksilber bzw. eine Änderung der Pulsfrequenz um 20 Schläge in der Minute betrachtet. In anderen Fällen wurde als Wirkung der Anästhesie erhebliche Blutdrucksteigerung, in einzelnen Fällen auch erhebliche Blutdrucksenkung festgestellt. Zunächst seien 23 Fälle erwähnt, bei denen die Injektion des Novokain-Suprarenins zu einer erheblichen Blutdrucksteigerung führte, und zwar bei Patienten mit sehr verschiedenem Allgemeinzustand und Lebensalter und verschiedenen Erkrankungen, teils handelte es sich dabei um einfachere, teils um kompliziertere Anästhesierungsverfahren. Einzelheiten ergeben sich aus der mitgeteilten Übersicht, die außer Angaben über Diagnose, Alter des Patienten, Art der Anästhesie, Menge des Anästhetikums, den vor Beginn der Anästhesierung festgestellten Blutdruckwert, den nach Beginn der Injektionen erreichten Höchstwert desselben und die Zeit, innerhalb deren nach 
Beginn der Injektion der Höchstwert erreicht wird, angibt. Aus den folgenden Spalten ergibt sich noch, ob der höchste Blutdruckwert während der Anästhesierung bzw. am Schluß derselben erreicht wurde, oder ob der Höhepunkt des Blutdruckanstiegs crst nach vollendeter Anästhesierung entweder in der Pause vor Operationsbeginn oder erst während der Operation eintrat. Die beiden letzten Spalten geben die Zeitdauer der Anästhesierung und die Art der Operatior an.

Übersicht.

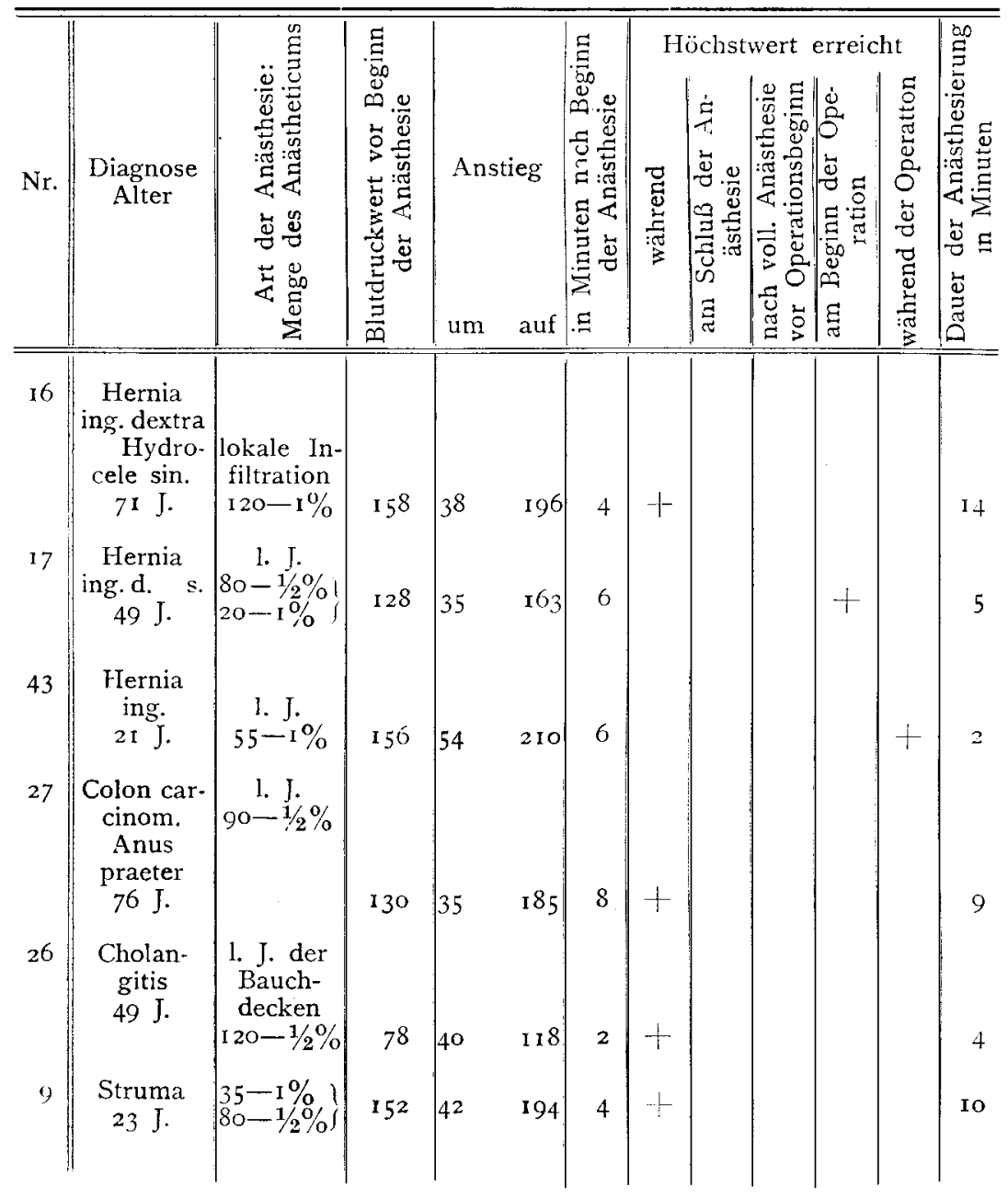




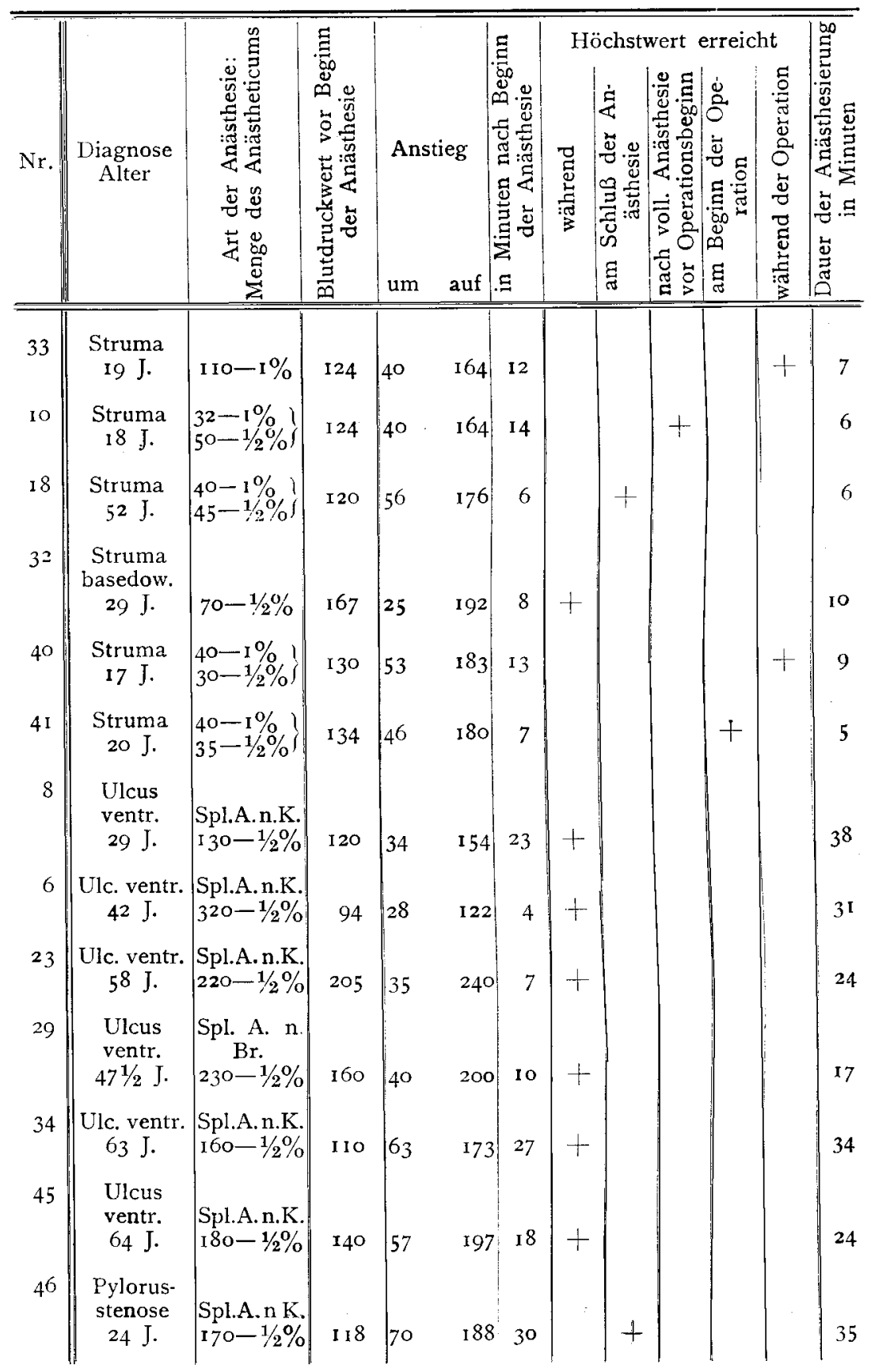




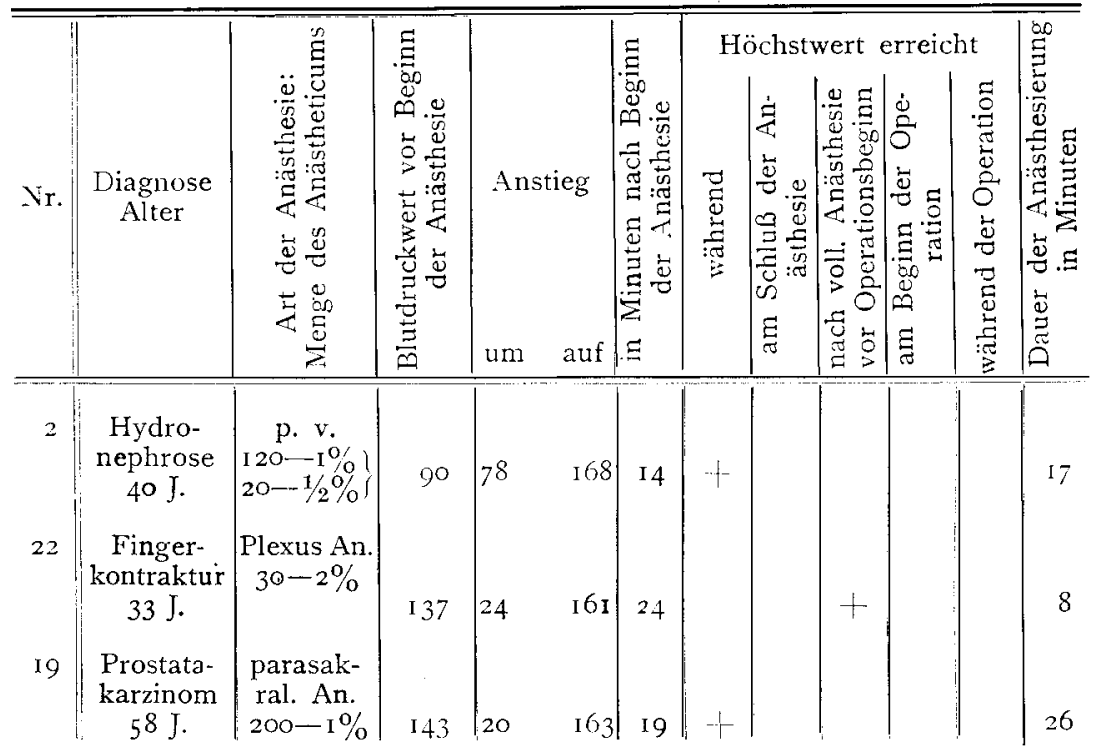

Nach dieser Ubersicht wurden im Anschlul3 an die NovokainSuprarenininjektionen Blutdrucksteigerungen um 2 I $-78 \mathrm{~mm} \mathrm{Hg}$ beobachtet, die Zeit, in der dieser Blutdruckanstieg erfolgte, betrug zwischen 2 und 30 Minuten nach Beginn der Anästhesierung. Als Beispiel eines raschen und beträchtlichen Blutdruckanstiegs sei Nr. 9 erwähnt, Blutdrucksteigerung von $152 \mathrm{~mm}$ auf $194 \mathrm{~mm} \mathrm{Hg}$ in 4 Minuten, ferner Nr. 4 I Steigerung von $134 \mathrm{~mm}$ auf $180 \mathrm{~mm}$ in 7 Minuten, Nr. 43 Blutdruckanstieg von 156 auf 210 mm (queck silber in 6 Minuten erwähnt. In einzelnen Fällen ist der Blutdruckanstieg in Wirklichkeit noch erheblicher, als die Differenz zwischen Höchstwert und letztem Wert vor Beginn der Injektion ergibt, wenn nämlich in den ersten Minuten nach Beginn der Anästhesierung erst eine Blutdrucksenkung erfolgt, z. B. bei Nr. I8 (I), wo zunächst der Blutdruck von $120 \mathrm{~mm}$ auf $106 \mathrm{~mm}$ abfiel und dann innerhalb der nächsten 2 Minuten von 106 auf 176 anstieg, ferner Nr. 23. wo der Blutdruck von $205 \mathrm{~mm}$ während der ersten 4 Minuten der Anästhesierung auf 166 fiel, um dann innerhalb der nächsten 3 Minuten von $166 \mathrm{~mm} \mathrm{Hg}$ auf $240 \mathrm{~mm}$ anzusteigen, auch bei Nr. 29 trat während der ersten 5 Minuten der Anästhe-

(1) Siche Kurve. S. 169 . 
sierung eine Blutdrucksenkung um $30 \mathrm{~mm}$ ein, um dann innerhalb der nächsten 4 Minuten einem Anstieg um $70 \mathrm{~mm}$ Hg Platz zu machen.

Demnach läßt sich unterscheiden zwischen Fällen, bei denen unter dem Einfluß der Novokain-Suprarenininjektionen nur Blutdrucksteigerung, mehr oder weniger rasch, eintritt und Fällen, bei denen der Blutdrucksteigerung erst eine Blutdrucksenkung in verschiedenem Grade vorangeht. Die Beobachtungen zeigen außerdem, daß es wichtig ist, die Blutdruckmessungen in kurzen Zeitabständen vorzunehmen, wenn nicht wesentliche Blutdruckschwan kungen unbemerkt bleiben sollen (I). Vielleicht erklären sich hieraus zum Teil die widersprechenden Angaben in der Literatur. Was die Zeit betrifft, innerhalb deren die Blutdrucksteigerung nach Beginn der Anästhesierung in Erscheinung tritt, so läßt sich ein verschiedenes Verhalten feststellen. In einzelnen Fällen (Nr. 9, I6, 26) ergibt die Blutdruckmessung bereits 2 Minuten nach Beginn der Injektion einen steilen Anstieg, in anderen Fällen tritt die Blutdruckerhöhung erst mehrere Minuten nach Beginn der Anästhesierung in Erscheinung, am häufigsten wurde sie 4-6 Minuten nach Beginn der ersten N.S.-Injektion feststellbar. Es kommt auch vor, daß die Blutdruckerhöhung erst mehrere Minuten nach Vollendung der Anästhesierung eintritt zu einer Zeit, wo der Patient unbehelligt auf dem Operationstisch liegt. Bei Nr. 23, 29, I 8 , wo zuerst eine Blutdrucksenkung erfolgte, trat der dann folgende Blutdruckanstieg ebenfalls 4-6 Minuten nach Beginn der Anästhesierung ein. Nr. 34 (II) bietet eine Besonderheit insofern, als

(I) Die Blutdruckwerte sind in Millimeter Quecksilber nach R iva $\mathrm{R}$ oc c i angegeben und beziehen sich auf den systolischen Druck. Auf den Originalkurven, die die Blutdruckkurve und die gleichzeitige Pulskurve wiedergeben, entsprach jeder Millimeter einer Zeit von 2 Minuten bzw. 2 mm Quecksilber. Da die Kurven in $3 / 4$ Größe der Originalkurve wiedergegeben sind, war es notwendig, die Blutdruckwerte stets in geraden Zahlen wiederzugeben, und zwar wurde immer die nächst höherc gerade Zahl genommen, dadurch entstehen gegenüber den Originalkurven ganz geringe Differenzen, die ohne Bedeutung sind. Die horizontal ausgezogenen schwarzen Striche geben Be ginn, Dauer und SchluB der Novokain-Suprarenininjektionen an, die punktierten horizontalen Linien, die Operationszeit.

(II) Siche Kurve, S. 169. 
die anfängliche Blutdrucksenkung I 4 Minuten anhielt, um dann in eine beträchtliche Blutdrucksteigerung überzugehen. Im allgemeinen scheint eine Erhöhung des Blutdrucks, wenn sie überhaupt durch die Anästhesierung ausgelöst wird, innerhalb der ersten Io Minuten nach Beginn derselben in Erscheinung zu treten, ein späterer Eintritt derselben selten zu sein, ein rascher Blutdruckanstieg innerhalb der ersten 2 Minuten nach Beginn der Lokalanästhesie häufiger vorzukommen. Der zeitlich sehr verschiedene Beginn der Blutdrucksteigerung spricht gegen eine Verursachung durch psychische Momente, und zwar sowohl in den. Fällen, bei dener die Blutdruckerhöhung erst in der Pause zwischen vollendeter Lokalanästhesie und Operationsbeginn eintritt, wo der Patient unter dem Einfluß der Morphiumwirkung sich zu seiner Umgebung teilnahmslos verhält, andererseits auch bei den Fällen, wo die Blutdruckerhöhung unmittelbar nach Beginn der Injektion in erbeblichem Grad eintritt. In den Fällen, wo nach dem ersten Einstich der Kanüle und Beginn der Injektion eine Wartezeit von mehreren Minuten eingeschaltet wurde, konnten wir wesentliche Schwankungen von Puls und Blutdruck innerhalb dieser Zeit nicht beobachten, auch bei der Splanchnicusanästhesie, wo das Tasten mit der Nadel in der Tiefe an der Wirbelsäule nicht immer ohne Unannehmlichkeiten für den Patienten vorübergeht, konnten wir einen wesentlichen Einfluß dieses Moments in der I'uls- und Blutdruckkurve nicht erkennen. Die vorstehenden Angaben beziehen sich allerdings auf ruhige und vernünftige Patienten, bei denen die Ausführung der Lokalanästhesie keinerlei Schwierigkeiten begegnete. Bei ängstlichen Patienten dürfte das psychische Verhalten nicht ohne Einfluß auf Puls und Blutdruck sein. Nr. 43 (I) ist besonders beachtenswert, da die Ausführung der Lokalanästhesie zur Hernienoperation nur 2 Minuten in Anspruch nahm und die Blutdrucksteigerung erst nach Vollendung der Anästhesie auftrat. Ein gleiches Verhalten konnte auch in anderen Fällen, wo die Dauer der Anästhesierung auch nur wenige Momente beanspruchte, beobachtet werden. Bei einzelnen Patienten fiel auch auf, wie wenig durch äußere Einflüsse, z. B. Lagerung von der rechten auf die linke Seite usw. Puls und Blutdruck beeinflußt

(I) Siehe: Kurve, S. 160 . 
wurden. Diese Fälle sprechen um so mehr dafür, die Erklärung der Blutdrucksteigerung in dem Adrenalingehalt der injizierten Lösung zu suchen. Tatsächlich entspricht auch zeitlicher Eintritt und Verlauf der Blutdrucksteigerung der bekannten Adrenalinwirkung, allerdings mit gewissen Einschränkungen, da es nicht angeht, die N.S.-Injektionen bei der Lokalanästhesie einfach mit einer subkutanen Suprareninjektion zu vergleichen. Dafür liegen die Verhältnisse bei der Lokalanästhesie viel komplizierter, es handelt sich nicht nur um eine andere Lösung, die äußer Suprarenin noch Novokain enthält, sondern auch um ganz anders zu bewertende Injektionen, die je nachdem, ob sie subkutan, intramuskulär, paravertebral usw. erfolgen, ganz andere Resorptionsbedingungen haben. Es handelt sich auch nicht um eine einmalige, sondern um mehrfache, über einen gewissen Zeitraum verteilte Injektionen, bei denen namentlich bei der Leitungsanästhesie rasch nacheinander an verschiedenen Stellen mehr oder weniger große N.S.-Depots angelegt werden. I) dung verschieden konzentrierter Lösungen bei dem gleichen Patienten werden die Verhältnisse ebenfalls anders als bei einer einmaligen Suprarenininjektion, ebenso auch durch die Pausen, die bei manchen Anästhesierungsverfahren zwischen den einzelnen Akten derselben nicht zu vermeiden sind.

Der zeitliche Eintritt der Blutdrucksteigerung und der Verlauf derselben entspricht in manchen Fällen der Wirkung der subkutanen Adrenalininjektion, in anderen Fällen wiederum tritt sie so rasch und plötzlich ein, wie es nach intravenöser Adrenalinzufuhr der Fall zu sein pflegt. Bezüglich der letzteren Fälle könnte man natürlich an die Injektion ganz geringer Mengen der N.S. Lösung in die Gefäßbahn denken, die ja vielleicht trotz sorgfältiger Injektionstechnik nach der Meinung mancher Autoren nicht vollkommen vermieden werden kann. Der Druck, unter dem die Injektion vorgenommen wird, dürfte auch hier nicht ohne Einfluß sein. Was den Grad der beobachteten Blutdrucksteigerung betrifft, so muß selbstverständlich berücksichtigt werden, daß in einzelnen Fällen, wo mit reichlichen Mengen konzentrierter Lösung gearbeitet wurde, die im ganzen injizierte Adrenalinmenge die sonst übliche Dosis von I $\mathrm{mg}$ um ein Vielfaches überschreitet. Auf die Bedeutung der Körpergegend, in der die Injektionen vor- 
genommen werden und ihren Einfluß auf die Resorptionswirkungen hier einzugehen, ist überflüssig.

Die in unseren Fällen beobachtete Blutdrucksteigerung pflegte sehr schnell wieder abzuklingen, meist trat 2-4 Minuten nach Erreichen des Höchstwertes wieder ein Blutdruckabfall ein, nur in wenigen Fällen blieb der erreichte hohe Blutdruck $12-15$ Minuten lang bestehen, um dann wieder abzufallen. Eine länger andauernde Blutdruckerhöhung über die Zeit der Anästhesierung hinaus, wie sie in der Literatur nach Adrenalininjektion bis zu cin- und zu mehrstündiger Dauer erwähnt wird, konnten wir in keinem einzigen Fall beobachten.

Den Fällen, bei denen der Blutdruckanstieg fast unmittelbar nach Beginn der Anästhesierung in steiler Kurve erfolgt, stehen andere gegenüber, bei denen die Blutdrucksteigerung langsamer und mit Unterbrechungen erfolgt, die einen Zusammenhang mit den einzelnen Abschnitten der Anästhesierung erkennen lassen, Als Beispiel sei die Kurve Nr. 46 (I) angeführt, wo bei einem 24 jährigen latienten die Splanchnicusanästhesie nach Kappis ausgeführt wurde. Aus dieser Kurve geht hervor, daß der Einstich der Kanüle und die Orientierung an der Wirbelsäule, deren Zeitpunkt lurch den ersten Punkt vor dem mit I bezeichneten horizontal ausgezogenen Strich angegeben ist, keine Blutdrucksteigerung auslöst, während im unmittelbaren Anschluß an die 5 Minuten später begonnene Injektion ins Splanchnicusgebiet rechts eine Blutdrucksteigerung von I $8 \mathrm{~mm}$ auf $166 \mathrm{~mm}$ folgt. Daran schließt sich unmittelbar wieder ein Blutdruckabfall an bis auf $140 \mathrm{~mm}$. Die Einführung der Kanüle, ebenfalls durch den zweiten P'unkt bezeichnet, auf der linken Seite läßt auch hier einen Einflu13 auf die Blutdruckkurve nicht erkennen, erst die 3 Minuten später vorgenommene Injektion ins linke Splanchnicusgebiet löst einen neuen Blutdruckanstieg aus von 140 auf $178 \mathrm{~mm}$, wobei dieser Wert für die nächsten 4 Minuten bestehen bleibt, um dann wieder auf $158 \mathrm{~mm}$ abzufallen. Hier beginnt dann unter dem Einfluß der Bauchdeckenanästhesie, allerdings nicht unmittelbar nach Beginn derselben, sondern erst 3 Minuten später ein neucr Blutdruckanstieg. der den höchsten Wert der ganzen Kurve mit I 88 mm

(I) Siche Kurve. S. 169. 
erreicht, um dann wieder abzufallen. In diesem Fall war auf die Ausführung der Anästhesie besonders lange Zeit verwandt worden, vom ersten Einstich der Nadel bis zur letzten Injektion im ganzen 35 Minuten, und zwischen den einzelnen Akten immer eine Pause von mehreren Minuten eingeschaltet, und man kann wohl den Eindruck gewinnen, daß es diesem Umstand, der langsamen Ausführung der Anästhesie, zuzuschreiben ist, daß der Blutdruckanstieg staffelweise erfolgte und sich über einen viel größeren Zeitraum verteilte, als wenn größere Mengen Novokain-Suprarenin an verschiedenen Körperstellen nacheinander ohne jede Pause injiziert werden.

Weiterhin sei die Blutdruckkurve von Nr.34 (Fig. I) angeführt, die sich ebenfalls auf eine Splanchnicusanästhesie nach Kappis bei einem 63 jährigen Patienten mit Magenulcus bezieht.

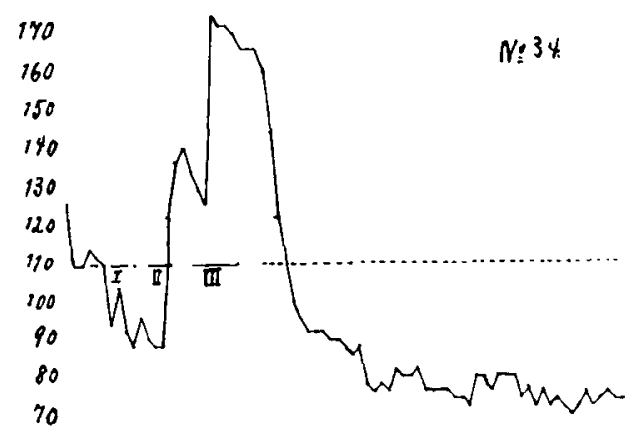

Kurve I.

V., 63 J. Ulcus ventriculi. Splanchnicusanästhesie nach Kappis. I. Recht $50 \mathrm{ccm} 1 / 2 \%$ N.S.L. II. Links $50 \mathrm{ccm} 1 / 2 \%$ N.S.L. III. Bauchdeckenanästhesie. $60 \mathrm{ccm} 1 / 2 \%$ N.S.L. Operation: Resektion.

Aus ihr geht hervor, daß der Blutdruck zunächst noch eine Neigung zum Abfallen zeigt, der Einstich der Kanüle zur Orientierung an der Wirbelsäule ist auch hier ohne Einfluß auf die Blutdruckkurve. Bei Beginn der Injektion auf der rechten Seite beträgt der Blutdruckwert $94 \mathrm{~mm}$, zeigt dann während der Injektion rechts einen geringen Anstieg auf $104 \mathrm{~mm}$, dann einen Abfall auf $88 \mathrm{~mm}$, wiederum einen geringen Anstieg auf $96 \mathrm{~mm}$, dann wieder Abfall auf $88 \mathrm{~mm}$. Unter dem Einfluß der Injektion auf beiden Seiten, jederseits $50 \mathrm{~mm} 1 / 2$ proz. N. S.-Lösung kommt also zunächst keine deutliche Blutdrucksteigerung zustande, im 
Klinische Untersuchungen über das Verhalten von Blutdruck usw. I6I

Gegenteil zeigt die Blutdruckkurve eine abfallende Tendenz. Erst am Schluß der Injektion auf der linken Seite kommt ein steiler Blutdruckanstieg zustande von $88 \mathrm{~mm}$ auf $\mathrm{I} 4 \mathrm{O} \mathrm{mm}$, der dann sofort wieder in eine geringe Blutdrucksenkung auf $126 \mathrm{~mm}$ übergeht. Hier schließt sich dann 4 Minuten nach Beginn der Bauchdeckenanästhesie ein neuer erheblicher Blutdruckanstieg an von $126 \mathrm{~mm}$ auf $174 \mathrm{~mm}$, danach beginnt dann der Blutdruck erst langsamer, dann rascher wieder abzufallen. Es fragt sich, worin die Erklärung für das Ausbleiben der Blutdrucksteigerung bzw. der Blutdrucksenkung während der Injektion der $100 \mathrm{ccm} \mathrm{N}$.- S.-Lösung ins Splanchnicusgebiet zu suchen ist, und warum eine deutliche Blutdrucksteigerung erst so spät eintrat. Es wäre wohl möglich, daß während der ersten Zeit der Anästhesierung an und für sich noch eine Neigung zum Blutdruckabfall bestanden hat, oder daß psychische Einflüsse für dieses Verhalten verantwortlich zu machen sind. Immerhin wäre ein derartig später Eintritt der ersten deutlichen Blutdrucksteigerung I 4 Minuten nach Beginn der ersten Injektion ein nach unseren Beobachtungen seltenes Vorkommen, eine besondere Unempfindlichkeit gegen Suprarenin kann bei dem Patienten wohl nicht angenommen werden, da die Erhöhung des Blutdrucks zwar spät, aber deutlich und in erheblichem Grade erfolgt. Daß die Injektion ins Splanchnicusgebiet an und für sich nicht blutdrucksenkend wirkt, wird noch besonders erwähnt werden. Die vorliegende Blutdruckkurve läßt aber noch eine andere Erklärungsmöglichkeit für die anfängliche Blutdrucksenkung und dem späten Eintritt der Blutdruckerhöhung zu, wenn man sich vergegenwärtigt, daß vom Suprarenin nicht blo $B$ eine blutdrucksteigernde, sondern auch eine blutdruckerniedrigende Wirkung wohl bekannt ist. Auf dies Verhalten des Blutdrucks nach Adrenalininjektion ist neuerdings wieder von $A$ rnstein und $\mathrm{Schle}$ sing e r hingewiesen worden, die von einer verkehrten Reaktion größerer Gefäßgebiete auf Adrenalinzufuhr sprechen, die zur Blutdrucksenkung führen kann. Experimentell gewonnene Tatsachen lassen eine erregende Wirkung des Adrenalins gleichzeitig auf Vasokonstriktoren und Vasodilatatoren annehmen, wodurch eine Blutdruckänderung sowohl im Sinne einer Steigerung als auch einer Senkung erklärlich wird, je nachdem der Tonus der Gefäßverengerer oder Gefäßerweiterer die Oberhand gewinnt. 
Diese Wirkungsmöglichkeit des Adrenalins wird zwar meist zur Erklärung der unter Umständen beträchtlichen Blutdrucksenkung herangezogen, die sich an die vorausgegangene Blutdrucksteigerung anschließt, es liegt aber kein Grund vor, sie nicht ebenfalls zur Erklärung einer Blutdrucksenkung zu benutzen, die einem folgenden Blutdruckanstieg vorausgeht. Weiterhin geht aus der Blutdruckkurve der rasche Übergang der Blutdrucksteigerung in Blutdrucksenkung hervor, der das erstemal zwischen Splanchnicusanästhesie und Bauchdeckenanästhesie etwas rascher erfolgt, als nach der zweiten Steigerung während der Bauchdeckenanästhesie. Das rasche Abklingen der blutdrucksteigernden Wirkung des Adrenalins ist bekannt, der Blutdruck kann bereits zu einer Zeit wieder sinken, wo im Kreislauf noch unzersetztes Adrenalin vorhanden ist. Bei der letzten Blutdrucksenkung von $174 \mathrm{~mm}$ abwärts ist die Tatsache beachtenswert, daß dieselbe bereits ihren Anfang nimmt zu einer Zeit, wo noch mehrere Minuten lang die Injektionen von Novokain-Suprarenin fortgesetzt werden. Es ist möglich, daß diese letzten Injektionen in ein Gebiet erfolgt sind, in dem durch die Wirkung der vorausgegangenen Injektionen der Blutkreislauf und damit auch die Resorption eine erhebliche Einschränkung erfahren hat. Es ist auch möglich, daß die Wirkungslosigkeit dieser letzten Injektionen auf einer mangelnden Anspruchsfähigkeit der Gefäßmuskulatur infolge der vorausgegangenen intensiven Reizung beruhen, wobei diese Erklärung im Gegensatz steht zu den Erfahrungen von Kretschmer, der bei wiederholter Adrenalinzufuhr immer neuen Blutdruckanstieg und bei dauernder Adrenalinzufuhr dauernde Blutdrucksteigerngen beobachten konnte. Seine Versuchsergebnisse werden vielfach gegen die Annahme einer Ermüdung der Gefäßsmuskulatur herangezogen, es dürften aber in dieser Beziehung die Versuchsergebnisse an Tieren nicht ohne weiteres auf den menschlichen Blutkreislauf, dessen Organe durch jahrzehntelange Beanspruchung und chronische Erkrankung in ihrer Funktionsbreite gelitten haben, zu übertragen sein.

Ferner sei die Blutdruckkurve von Nr. I6 angeführt, die sich auf einen 7 $\mathbf{I}$ jährigen Patienten mit rechtsseitiger Leistenhernie und linksseitiger Hydrocele bezieht. Die Anästhesie wurde auf der rechten Seite zuerst vorgenommen als lokale Infiltration und führte 
Klinische Untersuchungen über das Verhalten von Blutdruck usw. I63

zu einem raschen Blutdruckanstieg auf $196 \mathrm{~mm}$. Diese Steigerung erreicht mit dem Ende der Anästhesierung auf der rechten Seite ebenfalls ihr Ende. Von da ab beginnt dann ein Abfallen des Blutdrucks, das sich ohne Unterbrechung fortsetzt über die Zeit, in der auf der linken Seite die Anästhesierung zur Hydrocelenoperation vorgenommen wurde. Es ist also hier ein Beispiel dafür vorhanden, daß unter Umständen die Anlegung eines neuen Novokain-Suprarenindepots keine Blutdrucksteigerung auslöst, im Gegensatz zu anderen Beobachtungen (s. Kurve 46 Seite I69), wo die Anlegung eines neuen Novokain-Suprarenindepots einen neuen Blutdruckanstieg auslöst. Es gibt also im Verlauf der Blutdruckkurve Abschnitte, wo durch erneute Zufuhr adrenalinhaltiger Flüssigkeit eine Unterbrechung der Blutdrucksenkung nicht erreicht wird. Die angeführte Kurve ist zugleich ein Beispiel für den Verlauf der Blutdrucksenkung, die sich an die vorausgegangene Blut-

Kurve 2.

G., 7I J. Hernia ing. dext. + Hydrocele sin. Lokalanästhesie:

rechts $70-1 \%$ N.S.L.

links $50-1 \%$ N.S.L.

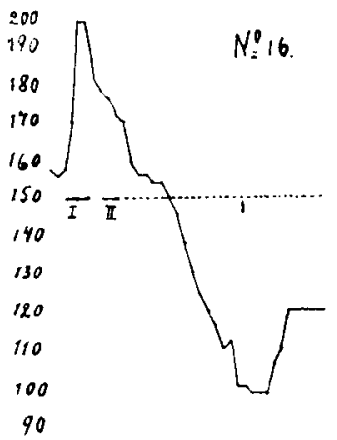

Drucksteigerung anschließt, und wie sie in yleicher Weise auch in anderen Fällen beobachtet werden konnte. Dieser abfallende Teil der Blutdruckkurve trat im allgemeinen in zwei Formen auf. Entweder war die Blutdrucksenkung beendet, wenn der Anfangswert wieder erreicht war und für den ferneren Verlauf während der Operation traten dann nur unwesentliche Schwankungen um diesen Wert herum auf. In anderen Fällen war der Verlauf der Blutdruckkurve so, daß nach vorausgegangener Blutdrucksteigerung der Abfall des Blutdrucks nach Wiedererreichen des Anfangswerts nicht beendet war, sondern sich eine weitere, in manchen Fällen sehr erhebliche Blutdrucksenkung tief unter den Anfangsswert anschloß. 
Vergleicht man bei Fällen, bei denen die Blutdruckschwankung nur im Anstieg auf den Höchstwert und Wiederabfall auf den Anfangswert besteht, die Zeitdauer dieser beiden Abschnitte, so ergeben sich folgende Werte:

Dauer der Blut- Dauer der Blut- Gesamtdauer der drucksteigerung. drucksenkung. Blutdruckschwan-

Nr. 17
,$\quad 43$
,,$\quad 27$
,, 32
,$" 40$
,, $4 I$

6 Min.
$6 "$
$8 "$
$8 "$
$13 "$
$7 "$

I2 Min.
$18, "$
7
$2 "$,
$20 "$,
$16 "$,
kung. I6 Min. 24 " 15, 10 , 33 " 23 ,

Es kommt also vor, daß der Blutdruckanstieg rascher erfolgt, als der Abfall und auch das umgekehrte Verhalten. Wie bereits erwähnt, kann das zeitliche Verhältnis des Eintritts des Höhepunkts der Blutdrucksteigerung zur Dauer der Anästhesierung verschieden sein, der Höhepunkt der Blutdrucksteigerung sowohl während der Anästhesierung als auch erst nach Vollendung derselben erreicht werden, dementsprechend kann auch der Zeitpunkt, bei dem die abfallende Blutdruckkurve wieder ihren Anfangswert erreicht noch in die Dauer der Anästhesierung fallen oder auch erst in die Zeit nach Vollendung derselben, je nach der Raschheit, mit der die Blutdruckschwankung verläuft; auch sehr hochgradige Blutdruckschwankungen können schon vor Beginn der Operaration vollkommen abgeklungen sein bei genügend langer Pause zwischen Schluß der Anästhesierung und Operationsbeginn. Umgekehrt konnte bei dem bereits erwähnten 2 I jährigen $\mathrm{Pa}$ tienten mit einer Leistenhernie, bei dem die Ausführung der Lokalanästhesie nur 2 Minuten in Anspruch nahm, 2 Minuten später die Operation sofort angeschlossen wurde, beobachtet werden, daß der Anfangswert des Blutdrucks erst mehrere Minuten nach beendeter Operation wieder erreicht wurde (s. Kurve Nr. 43). Die Einschaltung der allgemein vorgeschriebenen Wartezeit von Io Minuten nach Vollendung der Lokalanästhesie bis zu Operationsbeginn ist demnach nicht nur von Bedeutung für den Eintritt der Blutleere im Operationsgebiet, sondern auch für den Ablauf der allgemeinen Kreislaufwirkung der injizierten Novokain-Suprareninmenge insofern, als in manchen Fällen die erwähnten Blutdruckschwankungen 
Klinische Untersuchungen über das Verhalten von Blutdruck usw. 165

bei genügend langer Wartezeit vor Operationsbeginn vollendet sein können.

Anders zu bewerten sind dagegen die Erfahrungen bei den Fällen, wo die Blutdruckkurve nach erfolgtem Anstieg und Wiederabfall auf den Anfangswert nach Erreichung desselben weitersinkt bis zu Werten, die weit unter dem Anfangswert liegen, wie z. B. bei Nr. $1660 \mathrm{~mm}$, bei Nr. $1868 \mathrm{~mm}$, bei Nr. 23 Ior $\mathrm{mm}$ unter dem Anfangswert. Berücksichtigt man von den hierher grehörigen Fällen die Differenz zwischen dem Höhepunkt der Blutdruckkurve und dem tiefsten Blutdruckwert und die Zeit innerhalb deren diese Blutdrucksenkung verläuft, so ergeben sich beispielsweise folgende Werte:

Höchster Blut- Tiefster Blut- Differenz Zeitdauer der

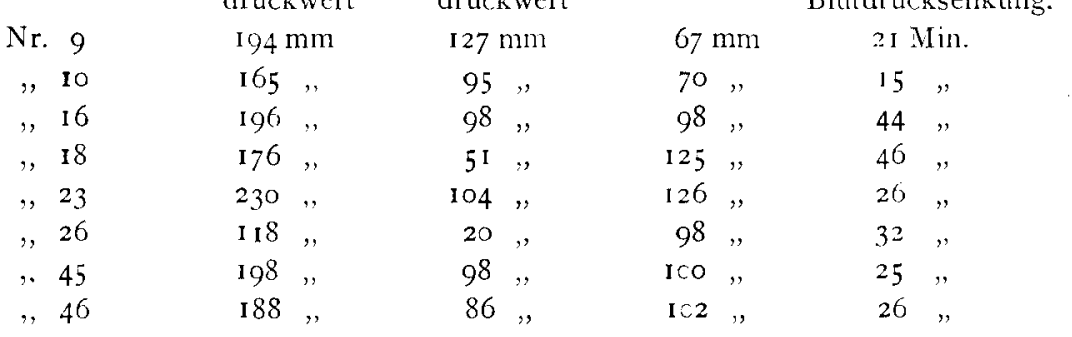

Diese Senkung vom höchsten zum tiefsten Wert findet teils ohne jede bzw. ohne wesentliche Unterbrechung statt, teils erleidet die Blutdrucksenkung in manchen Fällen nach Wiedercrreichen des Anfangswertes eine deutliche Unterbrechung, indem die Blutdruckkurve für einige Minuten auf dieser Höhe bleibt, manchmal auch einen unbedeutenden Anstieg erkennen läßt, um dann weiter bis zum tiefsten Punkt abzufallen. Es ist nicht der Zweck der vorliegenden Untersuchung, festzustellen, ob diese Blutdrucksenkungen allein auf dem Nachlassen der Adrenalinwirkung beruhen oder auf Rechnung der Novokainresorption zu setzen sind, jedenfalls bietet die Beurteilung dieses Abschnitts der Blutdruckkurve verschiedene Schwierigkeiten, da die Zeit der tiefsten Blutdrucksenkung in den seither beobachteten Fällen immer in die Operationszeit hineinfällt und damit neben der Wirkung des Novokain-Suprarenins auch die Einflüsse der Operation in Betracht kommen. Nur durch genaue graphische Darstellung der Blutdruckkurve und genaue Kontrolle der einzelnen Ereignisse, 
die als Ursache einer Blutdruckänderung in Betracht kommen können, ist es möglich, ein Urteil über die Ursachen der Blutdrucksenkung im einzelnen Fall zu gewinnen. Tritt die Senkung unter den Anfangswert bereits mehrere Minuten vor Operationsbeginn ein, so ist sie wenigstens für diese Zeit unabhängig von den Einflüssen der Operation. Bei der bereits erwähnten Kurve Nr. I6 ist der Anfangswert bereits 3 Minuten nach Beginn der Hernienoperation auf der rechten Seite erreicht, die Blutdrucksenkung unter den Anfangswert geht erheblich tiefer, um erst während der Operation der linksseitigen Hydrocele wieder in eine Blutdrucksteigerung überzugehen. Es ist wohl nicht möglich, in diesem Fall die Ursache der beobachteten Blutdrucksenkung in der in guter Anästhesie ausgeführten Operation nach Bassini zu finden, ein Operationsschok oder Blutverlust kommen in diesem Fall gar nicht in Frage, dagegen wohl die reine Resorptionswirkung des Novokain-Suprarenins und vielleicht auch die besondere Konstitution des Patienten. Bei der Kurve Nr. 45 (I), wo die Operation 3 Minuten nach Vollendung der Lokalanästhesie beginnt, zu einem Zeitpunkt, wo der abfallende Teil der Blutdruckkurve erst seinen Anfang genommen hat, ist der Anfangswert ca. Io Minuten nach Beginn der Operation erreicht gerade in dem Moment, in dem die Peritonealhöhle eröffnet wurde, so daß est wohl erlaubt ist, in diesem Falle in der Eröffnung der Bauchhöhle eine Ursache für die weitere Blutdrucksenkung zu suchen. Die Blutdruckkurve zeigt zwar nach Erreichen des tiefsten Wertes von $98 \mathrm{~mm}$ für die nächste Zeit wieder eine ansteigende Tendenz, während in der Bauchhöhle zahlreiche Verwachsungen teils stumpf, teils scharf in voller Schmerzlosigkeit gelöst wurden und wiederholt das Omentum minus gezerrt wurde, aber unmittelbar nach dem Schluß der Bauchhöhle steigt der Blutdruckwert wieder auf $134 \mathrm{~mm}$, um sich in dieser Höhe während der weiteren Bauchdeckennaht zu halten. Am Schluß der Operation ist der Blutdruckwert nur wenige Millimeter niedriger wie vor Beginn der Operation. Der Blutverlust kann nach unseren Beobachtungen nicht als Ursache der beobachteten Blutdrucksenkung in Betracht kommen, er ist bei normal verlaufender Operation so gering und erfolgt bei

(I) Siehe Kurve, S. 169. 
längerdauernder Operation so langsam, daß eine Blutdrucksenkung durch ihn nicht bewirkt wird, nur plötzlich auftretende Blutung aus Arterien und größeren Venen kommen hier in Betracht, auf deren Bedeutung bei Besprechung der einzelnen Fälle noch näher eingregangen werden soll. In den beiden vorgehend erwähnten Fällen war die Blutdrucksenkung vor Schluß der Operation bereits vollendet und der Blutdruck wieder während des letzten Teiles der Operation im Steigen begriffen, demgegenüber steht eine Erfahrung bei der Patientin Nr.23, bei der in Splanchnicusanästhesie nach Kappis eine Probelaparotomie ausgeführt wurde und alle Organe der oberen Bauchhöhle, Magen, Duodenum, Gallenblase, Pankreas freigelegt und revidiert wurden, und zwar in sehr guter Anästhesie. Während und nach der Lokalanästhesie traten keinerlei Nebenerscheinungen auf, kurz nach Beginn der Operation schlief die Patientin bis kurz vor Beendigung derselben. Der Blutdruck zeigte bei ihr unter dem Einfluß der Bauchdecken anästhesie ( $100 \mathrm{ccm} 1 / 2$ proz. N.S.-Lösung) eine mäßige Steigerung von $200 \mathrm{~mm}$ auf $230 \mathrm{~mm}$, um 4 Minuten nach Operationsbeginn wieder abzufallen. Während der ganzen weiteren Dauer der Operation, die 34 Minuten in Anspruch nahm, hielt die Blutdrucksenkung an, anfangs rascher, später etwas langsamer und mit kleinen Unterbrechungen. Am Schluß der Operation betrug der Blutdruck 1o8 mm, es hatte also eine sehr erhebliche Blutdruckerniedrigung stattgefunden, auch das übrige Aussehen der Patientin war nicht so, wie es sonst auch nach größeren, in Lokalanästhesie ausgeführten Bauchoperationen zu sein pflegt, die Blutdrucksenkung war aber, wie weitere Messungen ergaben, nicht mit dem Schluß der Operation vollendet, sondern dauerte noch $\mathrm{I} 1 / 4$ Stunde weiter an, bis auf $89 \mathrm{~mm}$ und erst am nächsten Tag betrug der Blutdruck wieder $160 \mathrm{~mm}$ und war immer noch weit unter dem am Tag vor der Operation festgestellten Wert.

Die Tatsache, daß im vorliegenden Falle die Blutdrucksenkung die Operationszeit weit überdauert hat, legt es nahe, auch noch nach einer anderen Ursache für dieses Verhalten außer der Anästhesie und den Einflüssen der Operation zu suchen. Beachtenswert erscheint in dieser Beziehung, daB die Patientin eine starke Arteriosklerose der Halsarterien und der ganzen Armarterien zeigte und der Blutdruck bei ihr schon normalerweise über 
$200 \mathrm{~mm} \mathrm{Hg}$ betrug. Arnstein und Schlesinger haben auf einen besonderen Zusammenhang von Arteriosklerosen und Blutdruckerniedrigung nach subkutaner Adrenalininjektion hingewiesen. Nach ihren Untersuchungen besteht bei älteren Patienten an und für sich eine gewisse Intoleranz gegen Adrenalin, insofern als auch bei geringen Adrenalinmengen unangenehme, unter Umständen bedrohliche Folgezustände bewirkt werden können im Gegensatz zu den Erfahrungen bei jüngeren Individuen. Was bei den letzteren nur ausnahmsweise beobachtet wurde, kam bei älteren Patienten in einem Drittel der untersuchten Fälle vor, daß nämlich der Blutdruck nach subkutaner Adrenalininjektion den normalen Anstieg vermissen ließ, entweder unverändert blieb oder nach geringer Erhöhung verschieden rasch abfiel, wobei die Blutdrucksenkung zuweilen erst nach $\mathrm{I}-2$ Stunden ihr Maximum erreichte und so erheblich war, daß bei einer Anzahl von Patienten ein Kollaps eintrat. Diese Blutdruckerniedrigung soll dann auf ein Nachlassen der Herzkraft zurückzuführen sein, wenn gleichzeitig ein rascher Puls, beschleunigte, oberflächliche Atmung, Schweißausbruch usw. bestehen. Als Erklärung kann aber auch eine verkehrte Reaktion größerer Gefäßgebiete auf Adrenalin herangezogen werden, wenn nämlich unter gewissen Umständen die gefäßerweiternde Wirkung des Adrenalins überwiegt. Nach Arnstein und $\mathrm{Schlesinger} \mathrm{kann} \mathrm{in} \mathrm{der} \mathrm{arteriosklerotischen} \mathrm{Er-}$ krankung der Gefäße die Ursache einer abnormen Reaktion der Gefäßmuskulatur im Sinne einer Gefäßerweiterung gesucht werden. In diesem Sinne lassen sich vielleicht auch die Erfahrungen verwerten, die man gelegentlich nach Lokalanästhesie bei älteren Patienten machen kann, daß nämlich trotz genügend langer Wartezeit eine Anämie im direkt infiltrierten Operationsgebiet nicht eintritt. Bei verschiedenen Patienten über 60 Jahre konnten wir auch feststellen, daß nach der Injektion größerer Mengen von Novokain-Suprarenin nicht Blutdrucksteigerung, sondern länger dauernde Blutdrucksenkung folgte(I). Es ist daher erklärlich, daß das Verhalten des Blutdrucks während und nach der Lokalanästhesie nicht einheitlich, sondern nach dem Zustand der Blutgefäße verschieden ist, wobei der Einfluß des Lebensalters und

(I) Siche Kurve, S. I69. 
Klinische Untersuchungen über das Verhalten von Blutdruck usw. I69
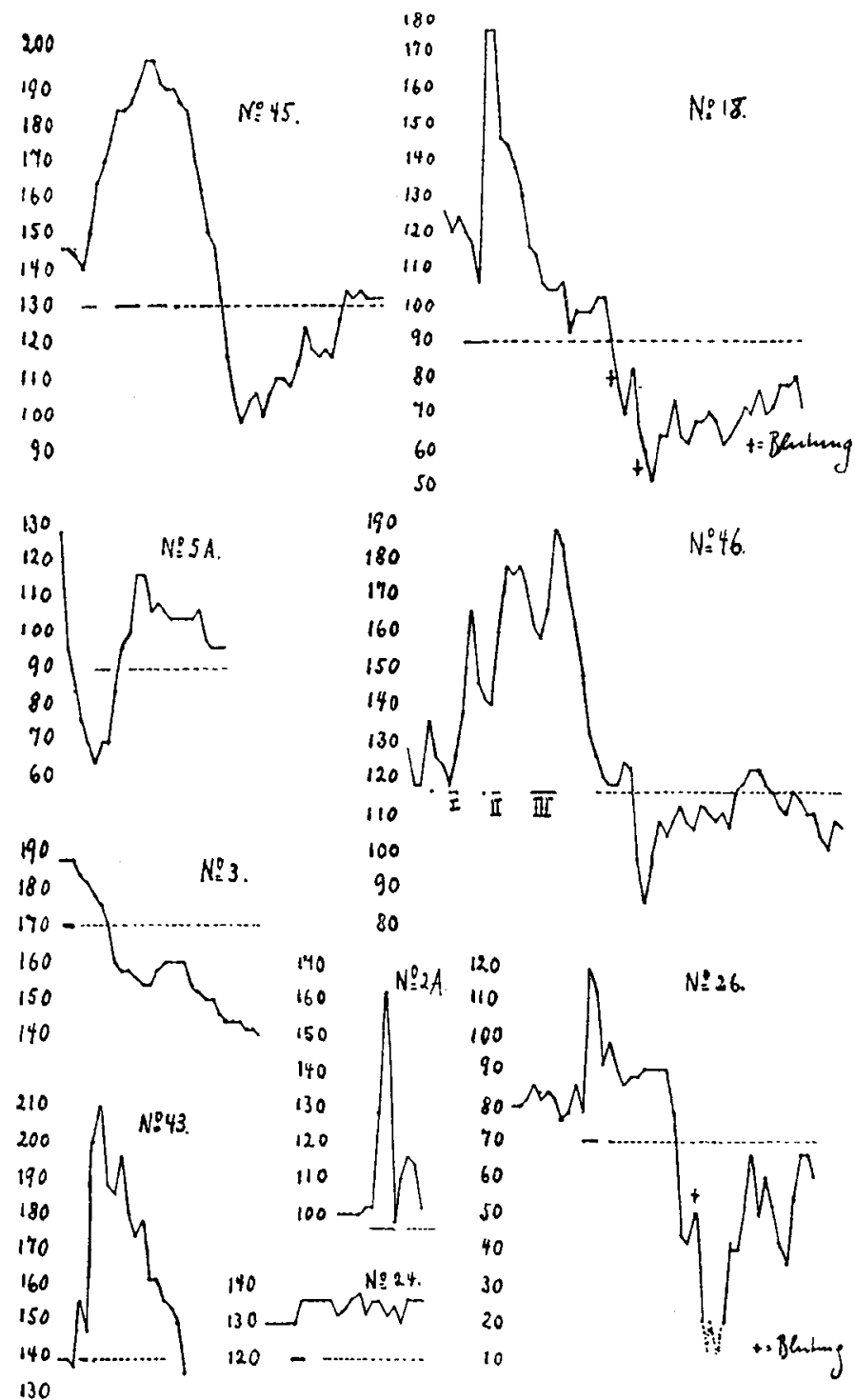

80

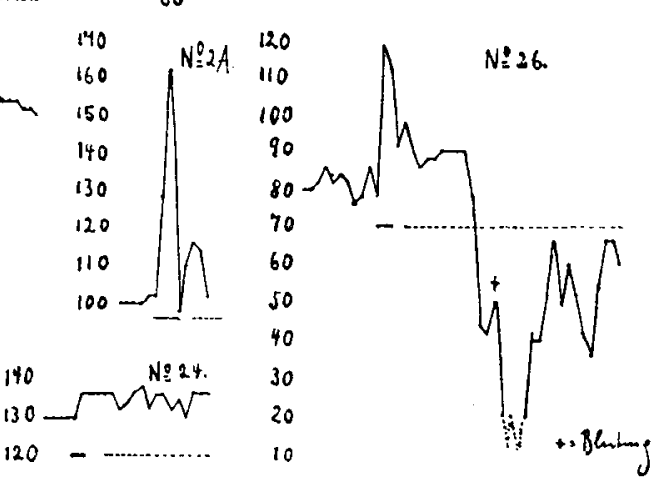

Kurve 3-II.

der in höheren Lebensjahren zunehmenden Arteriosklerose sich nur aus einer größeren Reihe von Beobachtungen ermitteln läßt. Vom praktischen Standpunkt aus muß mit der Möglichkeit einer erheblichen Blutdrucksenkung nach Novokain-Suprarenin- 
injektionen gerechnet werden, und damit erhebt sich die weitere praktisch wichtige Frage, ob diese Blutdrucksenkung, die nach unseren Erfahrungen vielfach schon vor Anfang der Operation beginnt, ihr Maximum während der Operation erreicht, einen gefährlichen Zustand bedeutet. Von diesem Gesichtspunkt aus seien hier 2 Fälle angeführt :

I. Nr. 18: $52 \mathrm{~J}$. Struma. (S. Blutdruckkurve Nr. 18.)

Vor Beginn der Anästhesierung betrug der Blutdruck I20 bis $125 \mathrm{~mm} \mathrm{Hg}$, während der ersten 4 Minuten der Anästhesierung (paravertebral 3. 4. Zervikainerv beiderseits je $20 \mathrm{ccm}$ ı proz. N. S.-Lösung, subkutan $45 \mathrm{ccm} 1 / 2$ proz. N.S.-L.) trat ein Sinken des Blutdrucks auf ro6 $\mathrm{mm}$ ein. Darauf folgte während der beiden letzten Minuten der Anästhesierung ein steiler Anstieg von $106 \mathrm{~mm}$ auf $176 \mathrm{~mm}$, dieser Höchstwert bestand 2 Minuten lang, daran schloß sich ein Wiederabfallen des Blutdrucks, der etwa il Minuten nach Beginn der Operation wieder den Anfangswert von I20 mm erreicht hatte. Auch weiterhin zeigte der Blutdruck noch die Neigung zum Fallen, wenn auch langsamer und mit geringen Unterbrechungen. 22 Minuten nach Beginn der Operation zeigte die Blutdruckkurve den Wert von $92 \mathrm{~mm}$, woran sich innerhalb der nächsten to Minuten ein langsames Ansteigen auf $102 \mathrm{~mm}$, dann wieder eine Senkung auf $90 \mathrm{~mm}$ anschloß. In diesem Moment trat bei der Unterbindung der Art. thyr. sup. durch Anreißen mehrerer Venen eine Blutung auf, die zwar durch Tamponade gleich gestillt wurde, aber doch zu einer Blutdruckscnkung von 90 auf $70 \mathrm{~mm}$ führte. In den nächsten beiden Minuten trat zunächst ein Blutdruckanstieg auf $82 \mathrm{~mm}$, dann wieder ein Abfallen auf $68 \mathrm{~mm}$ ein. In diesem Augenblick wurde die Tamponade weggenommen und die Blutung durch Fassen der Gefäße gestillt. Dabei führte die nach Wegnahme der Tamponade unvermeidliche Blutung zu einer weiteren Blutdrucksenkung von 68 auf $52 \mathrm{~mm}$. Erst nach der endgültigen Blutstillung zeigte die Blutdruckkurve wieder einen mit Unterbrechungen bis zum Schluß der Operation anhaltenden Anstieg bis auf $72 \mathrm{~mm}$. Der Schlußwert der Blutdruckkurve lag nur $48 \mathrm{~mm}$ unterhalb des Anfangswertes, die dazwischenliegende Blutdruckschwankung war aber viel beträchtlicher, sie umfaßte einen steilen Anstieg um $70 \mathrm{~mm}$ innerhalb 2 Minuten und eine erhebliche Blutdrucksenkung um $124 \mathrm{~mm}$ in 46 Minuten. Es unterliegt keinem Zweifel, daß namentlich an dem letzten Teil der Blutdrucksenkung auf den niedrigen Wert von $52 \mathrm{~mm}$ die erwähnte Blutung schuld ist. Außerdem ist wichtig, daß bei dem Pat. unmittelbar nach dem Einsetzen der Blutung auch Zeichen des Kollapses bestanden. Die Extremitäten fühlten sich kühl an, waren mit Schweiß bedeckt, das Aussehen blaß, der Puls klein und weich, aber nicht wesentlich beschleunigt. Dieser Zustand dauerte etwa ro Minuten lang und ging 
allmählich ohne besondere thcrapeutische Maßnahmen vorüber. Es handelt sich also hier um ein Bcispicl für einen Kollaps bei einer Strumaoperation in Lokalanästhesie, bei der die Hauptschuld an dem Zustandekommen des Kollapses der aufgetretenen Blutung beizumessen ist. Dabei ist allerdings zu berücksichtigen, daß in dem Moment, in dem die Blutung einsetzte, der Blutdruck bereits eine erhebliche Senkung von $176 \mathrm{~mm}$ auf $90 \mathrm{~mm}$ erfahren hatte, und damit auch die Frage naheliegend, ob etwa die Blutung, die an und für sich nicht besonders stark war, und bei zerreißlichen Kropfgefäßen öfters vorkommt, auch zum Kollaps geführt hätte, wenn sie einige Zeit vorher, wo der Blutdruck noch wesentlich höher war, eingetreten wäre. Eine Blutung ist natürlich bei schon vorhandener Kreislaufschwäche und niedrigem Blutdruck besonders gefährlich und der Verlust nur weniger Kubikzentimeter Blut kann in diesem $\mathrm{Zu}$ stand genügen, um einen Kollaps herbeizuführen. Im vorliegenden Fall hat für das Zustandekommen des Kollapses der Zufall insofern eine Rolle gespielt, als cine an und für sich nicht bedeutende Blutung eintrat in dem Moment, wo der Blutdruck infolge NovokainSuprareninresorption eine Senkung erfahren hatte. Auf das Zusam. mentreffen ähnlicher, blutdruckerniedrigender Momente während der Operation mit bereits bestehender Blutdrucksenkung als Folge der vorausgegangenen Novokain-Suprarenininjektion ist demnach besonder zu achten, im Vergleich zu den Fällen, die wir auch beobachten konnten, bei denen zeitweise eine starke Blutdrucksenkung während der Operation auftritt, aber bei ungestörtem Operationsverlauf ohne Kollapserscheinungen vorübergeht.

In diesem Zusammenhang ist ein weiterer Fall Nr.26 (s. Blutdruckkurve Nr.26), wichtig, der eine $49 \mathrm{j}$. Frau betrifft, die seit mehreren Jahren an Gallensteinkoliken litt und in sehr jämmerlichem Zustand zur Operation kam. Seit mehreren Tagen bestand Ikterus, hohes Fieber und Schüttelfröste, Erbrechen und ein aufgetriebener Bauch. Die Temperatur war über 39 Grad, der Puls an der Radialis schwach fühlbar, 149 Schläge in der Minute, der Blutdruck zwischen 75 und $86 \mathrm{~mm}$, die Zunge trocken und belegt, das Aussehen verfallen und peritonitisch. Der Allgemeinzustand war so schlecht, $\mathrm{da} B$ an eine Operation in Narkose nicht mchr gedacht werden konnte, deshalb wurde nach vorausgegangener Morphiuminjektion an beiden Rippenbogen die Leitungsanästhesie der Bauchwandnerven mit $120 \mathrm{ccm} 1 / 2$ proz. N. S.-Lösung ausgeführt. Sofort nach Beginn der Anästhesierung zeigte sich ein Blutdruckansticg ron $78 \mathrm{~mm}$ auf I $8 \mathrm{~mm}$, der sofort wieder noch vor Vollendung der Anästhesierung abzufallen begann. Bei Beginn der Operation betrug der Blutdruck $98 \mathrm{~mm}$, I6 Minuten später $90 \mathrm{~mm}$. Die Operation selbst war, da es sich nur um eine Bauchdeckenanästhesic handelte, nicht ganz schmerzlos, z. B. beim Abtamponicren und beim Vorzichen der Leber. 
Obwohl die Pat. während dieser schmerzhaften Momente etwas stöhnte und preßte, kam doch in der Blutdruckkurve nicht der geringste Anstieg zum Alusdruck. Die Eröffnung des Peritoneums, 4 Minuten nach Beginn der Operation, war ebenfalls ohne Einfluß auf die Blutdruckkurve, erst if Minuten nach Operationsbeginn, als die erwähnten schmerzhaften Akte schon vorüber waren, begann eine starke Blutdrucksenkung von $90 \mathrm{~mm}$ auf $42 \mathrm{~mm}$ innerhalb $6 \mathrm{Mi}$. nuten, wobei der Puls in dieser Zeit an der Radialis so schwach wurde, $\mathrm{da} B$ die weitere Zählung an der Carotis vorgenommen werden mußte. In den folgenden Minuten zeigte die Blutdruckkurve einen geringen Anstieg bis auf $50 \mathrm{~mm}$. In diesem Moment setzte eine Blutung aus der Art. cystica ein, die zwar zunächst durch Tamponade, dann durch Fassen mit der Klemme gestillt wurde und sicherlich nur zu einem Blutverlust von wenigen Kubikzentimetern geführt hatte, aber doch genügte um ein weiteres Sinken des Blutdrucks bis unter den Wert von $20 \mathrm{~mm}$, dem tiefsten Wert, der auf der Skala ablesbar war, herbeizuführen. Erst nach weiteren 6 Minuten erreichte der Blutdruck unter dem Einfluß von Kampfer, Adrenalin, intravenöser Infusion wieder einen meßbaren Wert und zeigte weiterhin einen Anstieg auf $66 \mathrm{~mm}$. Während des weiteren Operationsverlaufs traten zwar noch einige Blutdruckschwankungen auf, aber am SchluB der Operation betrug der Blutdruck doch $60 \mathrm{~mm}$, und war nicht wesentlich verschieden von dem Wert vor Beginn der Anästhesierung. Die Pat. hat sich während der nächsten Tage zunächst noch etwas erholt, kam aber dann doch noch zum Exitus. Auch hier kann der Lokalanästhesie als solcher nicht die Schuld für die Entstehung des Kollapses ge. geben werden, dieser war durch die Blutung bedingt, die, zwar unerheblich, wie im vorhergehenden Falle einsetztc, als der Blutdruck bereits einen abnorm niedrigen Wert erreicht hatte. Auch wenn man im vorliegenden Fall die Blutdrucksenkung bis zum Eintritt der Blutung auf Rechnung des Novokain-Suprarenins setzen will, so muß man dem doch gegenüber halten, daß die Pat. es nur der Lokalanästhesie zu verdanken hatte, daß sie lebend vom Operations. tisch kam.

Pa ul berichtet über einen schweren Kollaps bei einem Fall, der in seinem Verlauf Ähnlichkeit zeigt mit den hier mitgeteilten Erfahrungen.

Bei einer $22 \mathrm{j}$. Pat. in gutem Allgemeinzustand wurde die Splanchnicusanästhesie nach $\mathrm{K}$ a $\mathrm{p}$ p i s zwecks Vornahme der Gallenblasenoperation ausgeführt. Die Anästhesierung verlief bei der ruhigen und vernünftigen Pat. ohne Schwierigkeiten, die Anästhesie war ideal und erlaubte eine gute Orientierung in der Bauchhöhle. Bald nach der Injektion war der Puls auf I60 gestiegen, 30 Minuten nach der Injektion verfiel Pat. in tiefe Ohnmacht, das Gesicht wurde blaß, 
ganz zyanotisch, der Puls auffallend klein, die Atmung flach und aussetzend. Nach 5 Minuten ging dieser bedrohliche Zustand nach entsprechenden Maßnahmen vorüber und die Operation konnte in guter Anästhesie weitergeführt werden.

Auch in diesem Fall war während der Operation eine Blutung eingetreten durch Einreißen eines Astes des Vena portae, die sofort beherrscht wurde. Es bestand hier einmal eine Blutung, dann aber auch eine Kreislaufschwäche, wie sich aus der starken Pulsbeschleunigung entnehmen läßt. P a u 1 nimmt mit Recht an, daß der Kollaps nicht mit der Technik der Anästhesie in Zusammenhang gebracht werden kann, da er erst 30 Minuten nach Vollendung derselben auftrat, um so näher liegt die Annahme, daß der Zustand auf Kreislaufschwäche infolge Resorptionswirkung des Novokain-Suprarenins unter gleichzeitiger Mitwirkung der Blutung zurückzuführen ist. Auch wir müssen uns der Ansicht Pauls anschließen, daß die Lokalanästhesie unter Umständen bei scheinbar gesunden Leuten, bei denen eine Gegenindikation gegen eine Narkose nicht besteht, bedrohliche Erscheinungen hervorrufen kann, vor allem durch Blutdrucksenkung, die allerdings nach unseren Erfahrungen nicht ohne weiteres genügt, um einen Kollaps herbeizuführen, der erst dann auftritt, wie die beiden erwähnten Fälle zeigen, wenn zur Zeit der tiefen Blutdrucksenkung Einflüsse der Operation, geringfügige Blutungen und andere hinzukommen. Der Grad der Blutdrucksenkung dürfte nicht nur von der absoluten Menge und Konzentration den Anästheticums, dem Ort der Einführung abhängen, sondern auch von der Konstitution und Reaktionsfähigkeit des betreffenden Patienten und der Menge von Novokain-Suprarenin, die in der Zeiteinheit dem Organismus zugeführt wird, je nachdem die Anästhesierung langsam oder rasch ausgeführt wird. Die Resorptionswirkung des Novokain-Suprarenins führt unter Umständen zur Blutdrucksenkung und zeitweisen Kollapsgefahr, der Hinzutritt anderer blutdruckerniedrigender Momente zum wirklichen Kollaps.

Hier sind auch Ausführungen F in s t e r e r s bezüglich der Anwendung der Lokalanästhesie bei Peritonitis und Ileus von besonderem Interesse. Er erinnert daran, daß es bei beiden Erkrankungen infolge Vasomotorenschädigung zu einer Gefäßlähmung kommt, wobei die Blutdrucksenkung nicht voll zur Aus- 
bildung kommen kann, solange die gelähmten Bauchgefäße noch unter dem infolge des Meteorismus erhöhten intraabdominellen Druck stehen. Eine Narkose kann in solchen Fällen die bereits vorhandene Gefäßlähmung noch verstärken. Wenn nach Eröffnung der Bauchhöhle und erfolgter Eventration die Erhöhung des intraabdominalen Druckes fortfällt, so kann sich dann das Blut in die gelähmten, nicht mehr komprimierten Gefäße ohne Widerstand ergießen, und auf diese Weise ein Kollaps. entstehen, der auch durch die vorsichtigste Narkose nicht vermieden werden kann. Um auf die zwar gefährliche, aber im Interesse der Übersichtlichkeit der Operation doch wünschenswerte Eventration nicht verzichten zu müssen, geht $F$ in s te r e r so vor, daß die Eröffnung der Bauchhöhle in Lokalanästhesie vorgenommen wird und damit die Schädigung idurch die zur Peritonealeröffnung notwendig tiefe Narkose wegfällt. Zur weiteren Operation, insbesondere zur Eventration werden dann geringe Äthermengen gegeben, um den Blutdruck wieder zu heben. Finstere $r$ hält die grundsätzliche Durchführung derartiger Operationen in reiner Leitungsanästhesie, Splanchnicusanästhesie oder Lumbalanästhesie, unter Vermeidung auch der geringsten Äthermengen nicht für gerechtfertigt, wegen der Gefahr der Blutdrucksenkungen, die durch Resorption großer Novokainmengen bzw. rasche Resorption kleinerer Novokainmengen möglich sind. Er hat unter zahlreichen Fällen, die in Bauchdeckenanästhesie und anschließendem Ätherrausch operiert wurden, trotz Eventration der Baucheingeweide keinen bedrohlichen Kollaps gesehen.

Das Verhalten des Blutdrucks während und nach der Splanchnicusanästhesie verlangt insofern eine besondere Beachtung, als nach der Ansicht verschiedener Autoren ein größeres retroperitoneales Novokain-Suprarenindepot besonders intensive und unter Umständen auch gefährliche Wirkungen verursachen kann. Die unmittelbare Berührung der Splanchnicusäste mit der injizierten Lösung wird besonders betont; nach Buhre ist an die Möglichkeit einer unmittelbaren Beeinflussung der vasomotorischen Fasern der Splanchnici im Sinne einer Lähmung und folgender Blutdrucksenkung zu denken, wodurch die Gefahr einer Hirnanämie oder auch einer Herzschwäche infolge der plötzlichen Blutdruckschwankung gegeben wäre. Buhre vermutet, daß eine etwaige 
Vasomotorenlähmung im Splanchnicusgebiet durch die spezifische Wirkung des Adrenalins auf den Splanchnicus wieder ausgeglichen wird. Wir verfügen über 8 Fälle von Splanchnicusanästhesie nach Kappis, wo die genaue Kontrolle des Blutdrucks und Pulses ergibt, daß deren Verhalten nicht anders ist wie auch bei anderen Anästhesien. Bei 4 Fällen erfolgte auf die Injektion ins Splanchnicusgebiet eine Blutdrucksteigerung, in 3 Fällen eine geringe Blutdrucksenkung und dann ein Blutdruckanstieg, nur in einem Fall trat eine langsam verlaufende, aber nicht bedeutende Blutdrucksenkung ein. Auch das Verhalten des Pulses zeigte in diesen Fällen keine Besonderheiten. In 2 Fällen, wo die Splanchnicusanästhesie nach Braun ausgeführt wurde, erfolgte nach Injektion der vorgeschriebenen Ioo $\mathrm{ccm}$ auf die Mitte der Wirbelsäule eine deutliche Blutdrucksteigerung, die allmählich wieder abfiel. Nach unseren Beobachtungen können wir der Splanchnicusanästhesie eine besondere Stellung bezüglich etwaiger Blutdruckschwankungen nicht einräumen.

Bei verschiedenen Hernien und Hydrocelen waren die Schwankungen des Blutdrucks und der Pulsfrequenz sowohl während der Anästhesierung als auch während der Operation so unbedeutend, daß praktisch von einer Beeinflussung der Kreislauforgane weder durch die Anästhesie noch durch die Operation eine Rede sein konnte. Allerdings war in diesen Fällen auch die geringstmögliche Menge von Anästheticum in $1 / 2$ proz. Konzentration verwandt worden.

In 2 Fällen, bei denen die Plexusanästhesic nach $M$ ulley mit $30 \mathrm{ccm} 2$ proz. Lösung ausgeführt war, trat zwar eine deutliche, aber unerhebliche Blutdrucksteigerung ein, was wohl mit einer langsameren Resorption bei cndoneuraler Injektion zusammenhängcn mag.

Weiterhin ist bemerkenswert eine Pat. mit typischem Basedow, Exophthalmus, Tremor, doppelseitiger Struma, mäßiger psychischer Erregbarkeit, deren Herz aber kcinen krankhaften Befund bot. Die Lokalanästhesie (doppelseitige paravertebrale Injektion am 3. und 4. Zervikalnerv mit $50 \mathrm{ccm} 1 / 2$ proz. Lösung und subkutaner Injektion von $20 \mathrm{ccm} 1 / 2$ proz. Lösung) führte zu einem staffelförmigen Ansteigen der Blutdruckkurve um $25 \mathrm{~mm}$. Bei Beginn der Operation, I2 Minuten nach Vollendung der Anästhesie, war dic Blutdruckschwankung nach einer geringen Senkung unter den Anfangswert wieder ausgeglichen und während der ganzen folgenden Operation, Unterbindung sämtlicher 4 Arterien und doppelseitiger Resektion, zeigte der Blutdruck nur ganz geringe Schwankungen um den An- 
fangswert und hatte am Schluß der Operation den gleichen Wert wie vor Beginn der Anästhesierung. Die Pat. befand sich während der ganzen Operation in einem deutlich gesteigerten, wenn auch nicht gefährlichen Erregungszustand. Die Beeinflussung von Puls und Blutdruck durch Anästhesie und Operation war in diesem Fall von Basedow geringer als bei einer ganzen Anzahl von einfachen Strumen.

Diese Beobachtung entspricht auch wieder den bekannten Erfahrungen, wonach die Blutdruckwirkung des Adrenalins zeitlich unabhängig verläuft von den Wirkungen des Adrenalins auf andere Organe, im besonderen auch auf das Nervensystem. Wir haben unter zahlreichen Fällen mit Ausnahme der eben erwähnten Basedowpatientin trotz Anwendung hoher Adrenalinmengen in keinem einzigen Fall Erscheinungen gesehen, die als erhöhter Reizzustand im sympathischen Nervensystem infolge Adrenalinwirkung aufzufassen wären, auch dann nicht, wenn sehr erhebliche Blutdruckschwankungen zur Beobachtung kamen.

Für die Beurteilung der Blutdruckkurve ist natülich auch die gleichzeitige Pulskontrolle von großer Wichtigkeit. In unseren Fällen zeigten sich ebenfalls die in der Literatur als charakteristisch für Suprareninwirkung beobachteten Veränderungen der Pulsfrequenz, Beschleunigung, Verlangsamung, Unregelmäßigkeiten. Am häufigsten wurde Pulsbeschleunigung beobachtet, entweder gleich nach Beginn der Anästhesierung, manchmal auch erst später während der Operation. Die gleichzeitige Beobachtung von Blutdruck und Puls ergibt, daß das Verhalten des letzteren vielfach von dem Verlauf der Blutdruckkurve abhängig ist und auch hier ergeben sich weitgehende Analogien mit der Wirkung einer einfachen subkutanen Adrenalininjektion. In einer Anzahl von Fällen tritt die Veränderung der Pulsfrequenz nicht sofort nach Beginn der Anästhesierung, sondern erst einige Minuten später auf und ist dann nicht auf psychische Ursache zurückzuführen, sondern als Wirkung des resorbierten Novokain-Suprarenins aufzufassen. Zum Teil wurden erhebliche Pulsbeschleunigungen beobachtet; Vermehrung um 30-40 Schläge in der Minute, die rasch wieder vorübergingen, manchmal auch längere Zeit anhielten. Man kann rasch auftretende und wieder abklingende Pulsbeschleunigung beobachten, nach deren Rückgang erst ein Blutdruckanstieg erfólgt, so daß Pulsbeschleunigung und Blutdruckanstieg zeitlich nacheinander in Erscheinung treten. Andererseits geht auch ein 
deutlicher Blutdruckanstieg gleichzeitig mit einer deutlichen Pulsbeschleunigung einher, so daß die Pulskurve das gleiche Bild wie die Blutdruckkurve bietet und mit dem Sinken des Blutdrucks auch die Pulsbeschleunigung wieder verschwindet. In einzelnen Fällen tritt weder Blutdrucksteigerung noch Pulsbeschleunigung auf, in anderen Fällen kann man beobachten, daß zunächst eine deutliche Vermehrung der Pulsfrequenz sich entwickelt, während die Blutdrucksteigerung noch unerheblich ist und erst wenn die Blutdruckkurve steil in die Höhe geht. geht die Pulsbeschleunigung zurück und unter Umständen auch in Pulsverlangsamung über. Man gewinnt dann den Eindruck, daß die allgemeine Blutdrucksteigerung die Ursache der eintretenden Pulsverlangsamung ist. Dahin gehören auch die F älle, bei denen der Blutdruck nach Beginn der Anästhesierung sehr rasch und erheblich ansteigt, der Puls dagegen keine Beschleunigung erfährt oder von vornherein langsamer wird. Die Pulskurve kann dann das umgekehrte Bild bieten wie die Blutdruckkurve, solange der Blutdruck im Ansteigen begriffen ist, wird die Pulsfrequenz immer langsamer und erst wenn die Blutdruckkurve ihren Höhepunkt überschritten hat, und der Abfall derselben beginnt, setzt dann, zeitlich genau entsprechend, Pulsbeschleunigung ein, dem tiefsten Wert der Blutdruckkurve kann dann der höchste Wert der Pulskurve entsprechen.

Ebenso wichtig wie die Zählung der Pulsfrequenz ist die genaue Beobachtung der Qualität. Es läßt sich aus ihr unter gleichzeitiger Berücksichtigung des Blutdrucks und der Frequenz ein Urteil über die Becinflussung der Kreislauforgane während der einzelnen Abschnitte der Beobachtungszeit gewinnen. Man kann oft beobachten, daß in der Zeit des ersten Blutdruckanstiegs nach Beginn der Anästhesierung bei gleichzeitiger Pulsbeschleunigung. die Qualität des Pulses gut ist, er ist zwar beschleunigt, aber kräftig und gut gefüllt und damit besser als vor Beginn der Anästhesierung, so daß während dieser Periode infolge der Adrenalinwirkung Herz- und Blutgefäße zu einer höheren Leistung befähigt sind als vorher. Es entspricht dies Verhalten der Zeit, wo sich auch klinisch die vermehrte Herzarbeit durch starkes Pulsieren der großen Gefäße am Hals und an der Aorta und durch die sichtbare Verstärkung des Spitzenstoßes zu erkennen gibt. Die Lokalanästhesie hat dann die gesamten Kreislaufverhältnisse im 
günstigen Sinne beeinflußt. Diese Periode ist aber nach unseren Erfahrungen nur von kurzer Dauer entsprechend dem raschen Wiederabfallen des Blutdrucks. Auch wenn die direkte Beeinflussung der Herztätigkeit durch Adrenalinwirkung im Sinne einer Pulsbeschleunigung nicht in Erscheinung tritt, weil die gleichzeitige Blutdrucksteigerung die Vermehrung der Pulsfrequenz nicht zum Ausdruck kommen läßt oder sogar zur Pulsverlangsamung führt, so zeigt auch in diesen Fällen die Beschaffenheit des Pulses eine günstige Beeinflussung der Kreislauforgane. Man fühlt dann einen nicht beschleunigten oder sogar verlangsamten, aber gut gefüllten, kräftigen und gespannten Puls, der zeitweise die Beschaffenheit des Druckpulses zeigt. Es fragt sich weiterhin, ob im Gegensatz zu diesen Abschnitten der Puls- und Blutdruckkurve, wo wir von einer günstigen Wirkung der Lokalanästhesie auf die Kreislauforgane sprechen können, auch andere Abschnitte vorkommen, die auf eine ungünstige Beeinflussung derselben hindeuten. Diese Frage muß auf Grund verschiedener Einzelbeobachtungen bejaht werden, vor allem für die Abschnitte der Blutdruckkurve, wo eine länger dauernde erhebliche Blutdrucksenkung stattfindet, die durch die Operation als solche nicht ohne weiteres erklärt werden kann. Es können zwar bedeutende Senkungen des Blutdrucks sich entwickeln, ohne daß gleichzeitig eine wesentliche Änderung der Pulsfrequenz stattfindet. Die Qualität des Pulses wird aber doch in dieser Zeit deutlich weniger gut als vorher. Einfacher ist die Deutung in den Fällen, wo bei sinkendem Blutdruck der Puls immer mehr in die Höhe klettert und durch seine schlechte Füllung die eingetretene Kreislaufschwäche anzeigt. Man kann auch Fälle beobachten, bei denen gleichzeitig mit der Blutdrucksenkung die Pulsfrequenz immer langsamer und der Puls zugleich immer weicher und weniger kräftig wird, so daß man auch hier eine Verschlechterung der Kreislaufverhältnisse annehmen muß. Ob diese nun im einzelnen Fall auf eine mangelhafte Funktion der Blutgefäße oder auf eine Verringerung der Herzarbeit zurückzuführen ist, läßt sich nicht ohne weiteres ermitteln.

Bezüglich der sonstigen hier in Betracht kommenden Erscheinungen von seiten des Pulses sei auf die Untersuchungen von $\mathrm{S} \mathrm{ch}$ a $\mathrm{ps}$ verwiesen. 
Zur Ergänzung der vorstehenden Ausführungen seien noch folgende Blutdruckkurven angeführt:

Nr. 24 ${ }^{1}$ ). 43 j. Frau mit l. Leistenhernie. Lokalanästhesie mit $40 \mathrm{ccm} 1 / 2$ proz. N. S.-Lösung. Die Blutdruckschwankung während der Anästhesierung und während der Operationsdauer betrug noch nicht einmal Io $\mathrm{mm}$, die Schwankungen der Pulsfrequenz waren in diesem Fall auch ganz unbeträchtlich, eine merkbare Beeinflussung der Kreislauforgane hat überhaupt nicht stattgefunden.

Nr. $43^{1}$ ). 21 j. Mann mit r. Leistenhernie. Im Text crwähnt. Lokalanästhesie mit $55 \mathrm{ccm}$ I proz. N. S.-Lösung. Dauer der Anästhesierung 2 Minuten, im unmittelbaren Anschluß daran steiler Blutdruckanstieg. Die Blutdruckschwankung ist erst mehrere Minuten nach Schluß der Op. vollendet.

Nr. 3. 68 j. Mann mit einseitiger Leistenhernie. Lokalanästhesie mit $50 \mathrm{ccm}$ I proz. Lösung. Der Blutdruck zeigt im Anschluß an die Anästhesierung nur eine fallende Tendenz.

Nr. 2a ${ }^{1}$ ). 2 I j. Pat.mit Struma. Lokalanästhesie paravertebral 3. und 4. Zervikalnerv mit je $5 \mathrm{ccm}$ i proz. N. S.-L., subkutan $30 \mathrm{ccm} 1 / 2$ proz. N. S.-L. Sofort nach Beginn der Anästhesierung steiler Blutdruckanstieg, der am SchluB der Anästhesierung bereits wieder abgefallen ist. Die Kurve umfaßt nur die Zeit der Anästhesierung und die ersten Minuten der Op., weiterhin sind wesentliche Blutdruckschwankungen nicht aufgetreten.

Nr. $\left.8^{1}\right)$ ). 52 j. Mann, Struma. Lokalanästhesie paravertebral 3. und 4. Zervikalnerv beiderseits im ganzen $40 \mathrm{ccm}$ I proz. N.S.-Lösung, subkutan $45 \mathrm{ccm} 1 / 2$ proz. N.S.-Lösung. Kurve im Text erwähnt. Anfangs nach der Anästhesierung erst geringe Blutdrucksenkung, dann steiler Anstieg und Abfall unter den Anfangswert, Kollaps durch zweimalige Blutung. Schlußwert des Blutdrucks tief unter dem Anfangswert.

Nr. $\left.45^{1}\right) .64$ j. Pat., Ulcus ventriculi. Splanchnicusanästhesie nach $\mathrm{K}$ a p pis. Splanchnicus rechts $60 \mathrm{ccm} 1 / 2$ proz. N. S.-Lösung, Splanchnicus links $60 \mathrm{ccm} 1 / 2$ proz. N.S.-Lösung. Bauchdeckenanästhesie $60 \mathrm{ccm} 1 / 2$ proz. N. S.-Lösung. Mit Beginn der Anästhesierung sich langsam entwickelnder Blutdruckanstieg, dessen Abfall kurz vor Schluß der Anästhesierung beginnt. Senkung unter den Anfangswert, daran anschließend wieder allmähliche Blutdrucksteigerung, so da $B$ der Schlußwert fast gleich dem Anfangswert ist.

$\left.\mathrm{Nr} .4^{6}{ }^{1}\right) .24$ j. Pat. mit Adhäsionsbeschwerden und Pylorusstenose. Splanchnicusanästhesie nach Kap pis. Splanchnicus rechts $55 \mathrm{ccm}$ $1 / 2$ Proz., Splanchnicus links $55 \mathrm{ccm} 1 / 2$ Proz., Bauchdeckenanästhesie $60 \mathrm{ccm} 1 / 2$ proz. N.S.-Lösung. Staffelförmiger, langsamer Blutdruckanstieg, der mit Schluß der Anästhesierung wieder abfällt. Nach

1) Siehe Kurve S. I69. 
kurzer Unterbrechung Senkung unter den Anfangswert, dann wieder allmählige Steigerung. (Im Text erwähnt.)

Nr. 261). 49 j. Pat. Cholangitis. Bauchdeckenanästhesie mit $120 \mathrm{ccm} 1 / 2$ proz. N. S.-Lösung. Kurzdauernder Blutdruckanstieg, dann wieder Abfall weit unter den Anfangswert, Kollaps durch Hinzutreten einer Blutung.

Nr. 5a. 54 j. Pat. L. Leistenhernie. Lokalanästhesie $37 \mathrm{ccm}$ $1 / 2$ proz. N.S.-Lösung. Die Blutdruckkurve ist deshalb von besonderem Interesse, weil bereits vor Beginn der Anästhesierung eine starke und rasche Blutdrucksenkung exfolgte. In dieser Zeit bestand gleichzeitig Übelkeit, Brechreiz und Pulsverlangsamung. Es hat sich bei dem Pat. um eine Morphiumintoxikation gehandelt. Nach Vollendung der Anästhesie Blutdrucksteigerung. Die Kurve ist nicht $z$ l Ende geführt und zeigte nur noch unwesentliche Schwankungen. Diese Kurve zeigt zugleich den Wert einer zeitlich genauen Kontrolle der hier in Betracht kommenden Momente, sonst besteht die Gefahr, daß Nebenerscheinungen der Lokalanästhesie zur Last gelegt werden, die in Wirklichkeit mit ihr nichts zu tun haben.

Der Vergleich der einzelnen Kurven zeigt deutlich, daß es nicht angängig ist, aus dem Vergleich des Schlußwertes des Blutdrucks mit dem Anfangswert einen Rückschluß auf die Zwischenzeit zu ziehen. Die wesentlichen Schwankungen des Blutdrucks fallen in die Zeit der Anästhesierung und bei länger dauernden Operationen in die an die Anästhesierung sich unmittelbar anschließende Zeit. Nr. 45 und 46 geben ein Beispiel für Senkung des Blutdrucks unter den Anfangswert und allmähliches Wiederansteigen des Blutdrucks, so daß Anfangswert und Schlußwert nicht wesentlich verschieden sind. Nr. 18 und 26 zeigen den Einfluß einer hinzugetretenen Blutung in der Zeit, wo die Blutdruckkurve bereits einen niedrigen Stand erreicht hat.

\section{Literaturverzeichnis.}

I. Arnstein u. Schlesing er, Ungewöhnliche Wirkungen des Adrenalins im höheren Lebensalter. Wiener klin. Wochenschr. I9I9, Nr. 49, S. 1179 .

2. B a uer, Zur Funktionsprüfung des vegetativen Nervensystems. Arch. f. klin. Med., Bd. 107, S. 39 .

3. B a yer, Die normale und pathologische Physiologie des chromaffinen Gewebes der Nebennieren. (Erg. d. Allg. Path. u. path. Anat. d. Men.

I) Siche Kurve S. 169 . 
Klinische Untersuchungen über das Verhalten von Blutdruck usw. I8I

scben u. d. Tiere, 1910, 14. Jahrg, 2. Abtlg.) Enthält mit Nr. 57 I die bis dahin erschienene Literatur über Adrenalin.

4. B r a u n, Die Lokalanästhesie.

5. D enk, Die Gefahren und Schäden der Lokal-und Leitungsanästhesie. Ref. mit ausführlichem Literaturverzeichnis. Wiener klin. Wochenschr. I920, Nr. 29.

6. Finsterer, Der EinfluB der Lokalanästhesic auf die Indikations. stellung und dic Operationserfolge. Med. Klinik I920, Nr. 25-26.

7. Härtel, Dic Lokalanästhesic.

8. Ka uert, Uber die Anwendung des synthetischen Suprarenins in der inneren Medizin. Arch. f. klin. Med., Bd. Ioo, S. 387.

9. P a ul, Zur Splanchnicusanästhesie. Wiener klin. Wochenschr. I920, Nr. 24.

Io. Schaps, Nebenerscheinungen bei Lokalanästhesie mit Novokain. Suprarenin. Deutsche Zcitschr. f. Chir. Bd. 159, 'H. I, S. I. 